MEDLINE, EMBASE, Cochrane Library, Scopus, CINAHL, HTA and DARE, 3 websites and 7 trial registers were searched from 2008 to 2021. Study inclusion criteria were: malignant biliary obstruction; intervention as endoscopic RFA, either to fit a stent (primary RFA) or to clear a blocked stent (secondary RFA); primary outcomes were survival, quality of life or procedure-related adverse events. Risk of bias was assessed using the RoB 2.0 and ROBINS-I tools. Primary analysis was meta-analysis of the hazard ratio of mortality.

Results 68 studies (1742 patients) were identified but only 2 randomised trials, 1 retrospective case control study and 3 retrospective cohort studies reported a hazard ratio of death for primary RFA compared to stent-only control. The pooled hazard ratio of mortality for primary RFA compared to stent-only was 0.34 (95\% confidence interval (CI) 0.21 to 0.55$)$. There was moderate heterogeneity $\left(\mathrm{I}^{2}=53 \%\right)$ however the studies were consistently in favour of primary RFA. There was insufficient evidence available to analyse effectiveness in secondary RFA. No evidence about the impact on quality of life was found. There was no evidence of increased risk of cholangitis (risk ratio $1.15,95 \%$ CI 0.63 to 2.12 ) or pancreatitis (risk ratio $1.34,95 \% \mathrm{CI} 0.55$ to 3.25 ), but there was an increase in cholecystitis (risk ratio 11.47, 95\% CI 2.28 to 57.66). Inconsistencies in standard reporting and study design were noted e.g. adverse outcomes and lack of standardised comparator groups. RFA was estimated to cost $£ 2,659$ and produced 0.18 QALYs more than no RFA on average. With an ICER of $£ 14,392 /$ QALY, RFA was likely to be cost-effective at a threshold of $£ 20,000 /$ QALY. The source of the vast majority of decision uncertainty lay in the effect of RFA on stent patency.

Conclusions Primary RFA is associated with increased survival and appears cost-effective. The evidence for the impact of secondary RFA on survival and of quality of life is limited. There was no increase in the risk of post-ERCP cholangitis or pancreatitis but increased risk of cholecystitis. High quality RCTs to investigate primary and secondary RFA are needed with accurate documentation of quality of life, adverse event rates and survival.

\section{PTU-36 GASTROINTESTINAL BLEEDING EVENTS IN PATIENTS WITH A LEFT VENTRICULAR ASSIST DEVICE: A 10 YEAR EXPERIENCE}

${ }^{1}$ Ravi Ranjan*, 'Melanie Gunn, ${ }^{2}$ Guy MacGowan, ${ }^{1}$ John Leeds. ${ }^{1}$ Department of Gastroenterology, Newcastle Upon Tyne Teaching Hospitals, Newcastle Upon Tyne, UK; ${ }^{2}$ Department of Cardiology, Newcastle Upon Tyne Teaching Hospitals, Newcastle Upon Tyne, UK

\subsection{6/gutjnl-2021-BSG.109}

Introduction The advent of left ventricular assist devices (LVAD) has improved the survival and quality of life in patients with end stage heart failure however gastrointestinal bleeding remains one of the limitations. Little is known regarding important endoscopic findings and therapy rates.

Patients and methods A retrospective analysis of the LVAD database was conducted over the period 2009 - 2019. Data was collected on demographics, date of LVAD implantation, underlying cardiac diagnosis, history of anaemia, duration of implantation and survival. Each LVAD patient was cross checked on the endoscopy recording software for whether they had had any procedures, when, what type and findings. Univariate and multivariable analysis was performed.
Results 235 patients were included (median age 61.1 years, 203 males) with a median time of implantation of 465 days. Overall 56/235 (23.8\%) had undergone gastroscopy mainly for bleeding symptoms, of which 8/235 (3.4\%) were therapeutic. Multivariable analysis showed that undergoing gastroscopy was associated with a history of anaemia (adj OR 64.2, 95\% CI 22.5 - 182.9, p<0.0001), female sex (adj OR 6.84, 95\% CI $1.82-25.7, \mathrm{p}<0.0001)$ and aetiology of ischaemic heart disease (adj OR 3.05, 95\% CI 1.16 - 8.00, $\mathrm{p}=0.0235$ ). Therapeutic endoscopy was associated with increasing age (adj OR 1.09 , 95\% CI $0.99-1.19, \mathrm{p}=0.055)$ and aetiology of congenital heart disease (adj OR 12.9, 95\% CI 1.44 - 116.7, $\mathrm{p}=0.022$ ). Need for gastroscopy was not associated with survival however cumulative frequency plotting showed that $50 \%$ of gastroscopies occurred within 6 months of device implantation.

Conclusions Gastrointestinal bleeding symptoms are common in patients with LVAD however significant yield at gastroscopy is low. Highest yield is found in older patients and those with congenital heart disease as the aetiology.

\section{PTU-37 TO SCOPE OR NOT TO SCOPE: OUTCOMES OF ENDOSCOPY SURVEILLANCE IN OLDER ADULTS}

${ }^{1}$ Hoda Amar*, ${ }^{1}$ Mohamed Assal, ${ }^{2}$ Gui Han Lee, ${ }^{1}$ Katie Schwab. 'University Hospitals Dorset, Bournemouth, UK; ${ }^{2}$ University Hospital Southampton, Southampton, UK

\subsection{6/gutjnl-2021-BSG.110}

Introduction Updated guidance from the British Society of Gastroenterology (BSG) no longer recommends endoscopic surveillance after colorectal cancer resection or polypectomy in patients over 75 years. We aimed to evaluate the outcomes of surveillance in older adults in our local population, which is considered one of the most elderly in the country.

Methods A retrospective analysis of patient records was conducted for patients over 70 years, who had undergone colorectal cancer surgery with curative intent, between 2014 and 2016 at our district general hospital. We identified patients that had surveillance and those that did not. In the surveillance group, endoscopic findings were noted, including the presence of high-risk findings according to the BSG criteria, as well as complications following endoscopy. Parameters of interest for both groups were age, sex, ASA grade, Charlson comorbidity index (CCI), original tumour site, resection margin, TNM stage, CEA level, whether the patient received neoadjuvant or adjuvant therapy, overall survival and cause of death. Statistical analysis was performed using SPSS v27.

Results 207 patients were included in the study. 199 patients had major resection and 8 had endoscopic mucosal excision for their primary cancer. Median age was 77 years. Further demographics are shown in table 1. 108 patients had at least one surveillance endoscopy, of which 41 (38\%) identified polyps, including $11(10 \%)$ with high risk findings. No major complications were reported. Overall survival was greater in the surveillance group at 38 months, compared to 21.5 months in the non-surveillance group $(\mathrm{p}<0.01)$. Mortality due to colorectal cancer was lower in the surveillance group (8 patients vs 29 patients) including mortality due to local recurrence (1 patient vs 7 patients). Parameters that were significantly lower in the surveillance group were age, ASA grade, CCI, $M$ stage and CEA. There was no significant difference in sex, tumour site, resection margin, $\mathrm{T}$ stage, $\mathrm{N}$ stage and 